\title{
F-RESOLVABLE SPACES
}

\author{
INTISSAR DAHANE, LOBNA DRIDI AND SAMI LAZAAR
}

Abstract. In this paper we study topological spaces whose $\mathbf{F}$-reflections are resolvable. In particular, the reflectors $\mathbf{T}_{0}, \mathbf{S}, \boldsymbol{\rho}$, and $\mathbf{F H}$-reflection are characterized.

\section{INTRODUCTION}

In 1943, Hewitt introduced resolvable spaces. A topological space is called resolvable if it has two disjoints dense subsets, or equivalently if it has a subset $A$ such that $A$ and $X \backslash A$ are dense. By maximally irresolvable spaces, Hewitt means spaces such that any dense subset is open.

Recently, Arhangel'skii, Belaid, Dridi, and Lazaar have been concerned with maximally irresolvable spaces, which are also called submaximal spaces (for more information see [10] and [2]).

In [1], Belaid and Al-Hajri characterize topological spaces $X$ such that $\mathbf{K}(X)$ is resolvable in particular cases of the one-point compactification and the Wallman compactification. Furthermore, in [3] and [4] ([6] and [7]), the authors give a characterization of spaces such that their $\mathbf{F}$-reflections (their compactifications) are submaximal, door and nodec. In this paper $\mathbf{F}$ designates a covariant functor from the category Top to itself. So, it is natural to ask the same question for a resolvable space. That is characterize topological spaces such that their F-reflections are resolvable.

Firstly, let us recall the standard notion of reflective subcategories. A full subcategory $\mathcal{A}$ of $\mathcal{B}$ is called reflective with reflector $\mathbf{F}$ if and only if the embedding functor $\mathbf{I}$ from $\mathcal{A}$ to $\mathcal{B}$ has a left adjoint $\mathbf{F}: \mathcal{B} \longrightarrow \mathcal{A}$. Further, it is well known that for all $i \in\left\{0,1,2,3,3 \frac{1}{2}\right\}$ the subcategory $\mathbf{T o p}_{i}$ whose objects are $\mathbf{T}_{i}$-spaces is reflective in the category Top of all topological spaces.

It is with Belaid, Echi, and Lazaar in 2004 that we discovered some new separation axioms namely $\mathbf{T}_{(i, j)}$-spaces as follow (see [5]):

A topological space $X$ is called $\mathbf{T}_{(i, j)}$-space if and only if its $\mathbf{T}_{i}$-reflection is a $\mathbf{T}_{j}$ space. More generally, if we consider a reflector $\mathbf{F}$ from Top to itself and a topological property $\mathbf{P}, X$ is called $\mathbf{T}_{(\mathbf{F}, \mathbf{P})}$ if and only if its reflection $\mathbf{F}(X)$ satisfies the property $\mathbf{P}$. As a motivation of the foundation of these new separation axioms, some authors have been interested in particular cases, on the one hand, by topological properties such as door, submaximal, nodec and, on the other hand,

$M S C$ (2010): primary 54B30, 54D10, 46M15.

Keywords: categories, functors, resolvable spaces. 
by functors such as $\mathbf{T}_{0}, \mathbf{S}, \boldsymbol{\rho}$, and $\mathbf{F H}$-reflection. In this paper, we have interested by the notion of resolvable spaces as a topological property.

In the first section of this paper, we consider $\mathbf{T}_{0}$-reflection and the $\mathbf{S}$-reflection (called also soberification). Topological spaces whose $\mathbf{T}_{0}$-reflections ( $\mathbf{S}$-reflections) are resolvable are called characterized.

The second section is devoted to the same topics using $\boldsymbol{\rho}$-reflector and $\mathbf{F H}$ reflector.

\section{2. $\mathbf{T}_{0}$-Resolvable spaces and $\mathbf{S}$-Resolvable SPaCeS}

First let us recall the $\mathbf{T}_{0}$-reflection of a topolgical space. Let $X$ be a topological space. We define the binary relation $\sim$ on $X$ by $x \sim y$ if and only if $\overline{\{x\}}=\overline{\{y\}}$. Then, $\sim$ is an equivalence relation on $X$ and the resulting quotient space $\mathbf{T}_{0}(X):=$ $X / \sim$ is the $\mathbf{T}_{0}$-reflection of $X$.

The canonical surjection $\mu_{X}: X \longrightarrow \mathbf{T}_{0}(X)$ is a quasihomeomorphism (a continuous map $q: X \longrightarrow Y$ is said to be a quasihomeomorphism if $U \longmapsto q^{-1}(U)$ $\left(C \longmapsto q^{-1}(C)\right)$ defines a bijection $\mathcal{O}(Y) \longrightarrow \mathcal{O}(X)(\mathcal{F}(Y) \longrightarrow \mathcal{F}(X))$, where $\mathcal{O}(X)$ $(\mathcal{F}(X))$ is the collection of all open sets (closed sets of $X)$, see [9]).

Before giving the main result of this section let us introduce some definitions, notations and remarks.

Notations 2.1. [6, Notations 2.2] Let $X$ be a topological space, $a \in X$ and $A \subseteq X$. We denote:

(1) $d_{0}(a):=\{x \in X: \overline{\{x\}}=\overline{\{a\}}\}$.

(2) $d_{0}(A):=\cup\left[d_{0}(a) ; a \in A\right]$.

Remarks 2.2. $[6$, Remarks 2.3$]$ Let $X$ be a topological space and $A$ be a subset of $X$. The following properties hold.

(i) $d_{0}(A)=\mu_{X}^{-1}\left(\mu_{X}(A)\right)$.

(ii) $d_{0}\left(d_{0}(A)\right)=d_{0}(A)$.

(iii) $A \subseteq d_{0}(A) \subseteq \bar{A}$ and consequently $\overline{d_{0}(A)}=\bar{A}$.

(iv) In particular if $A$ is open (closed), then $d_{0}(A)=A$.

Definition 2.3. Let $X$ be a topological space. $X$ is called a $\boldsymbol{T}_{0}$-resolvable space if its $\mathbf{T}_{0}$-reflection is a resolvable space.

Now we are in a position to give the characterization of a $\mathbf{T}_{0}$-resolvable space.

Theorem 2.4. Let $X$ be a topological space. Then, the following statements are equivalent:

(1) $X$ is a $\boldsymbol{T}_{0}$-resolvable space;

(2) There exist two dense subsets $A_{1}$ and $A_{2}$ of $X$ satisfying $d_{0}\left(A_{1}\right) \cap d_{0}\left(A_{2}\right)=$ $\emptyset$.

Proof. We need a Lemma:

Lemma 2.5. [6, Lemma 2.16] Let $f: X \longrightarrow Y$ be a quasihomeomorphism. Then, the following statements are equivalent:

(1) $f$ is onto;

(2) For any subset $A$ of $Y$, we have $f^{-1}(\bar{A})=\overline{f^{-1}(A)}$. 
Proof of the Theorem:

$(1) \Longrightarrow(2)$

Suppose that $X$ is a $\mathbf{T}_{0}$-resolvable space. Then, there exist two subsets $A_{1}$ and $A_{2}$ of $X$ such that $\mu_{X}\left(A_{1}\right)$ and $\mu_{X}\left(A_{2}\right)$ are two disjoint dense subsets of $\mathbf{T}_{0}(X)$. So applying $\mu_{X}^{-1}$, one can see easily that $d_{0}\left(A_{1}\right)$ and $d_{0}\left(A_{2}\right)$ are disjoint. Now since $\mu_{X}$ is an onto quasihomeomorphism, Lemma 2.5 shows that $\mu_{X}^{-1}\left(\overline{\mu_{X}\left(A_{i}\right)}\right)=$ $\overline{\mu_{X}^{-1}\left(\mu_{X}\left(A_{i}\right)\right)}$ for every $i \in\{1,2\}$ and, consequently, $d_{0}\left(A_{1}\right)$ and $d_{0}\left(A_{2}\right)$ are two dense subsets in $X$, which means exactly that $A_{1}$ and $A_{2}$ are dense subsets in $X$ (see Remarks 2.2(iii)). Finally, $A_{1}$ and $A_{2}$ are two dense subsets of $X$ satisfying $d_{0}\left(A_{1}\right) \cap d_{0}\left(A_{2}\right)=\emptyset$.

$(2) \Longrightarrow(1)$

Conversely, let $A_{1}$ and $A_{2}$ be two dense subsets of $X$ such that $d_{0}\left(A_{1}\right) \cap d_{0}\left(A_{2}\right)=$ $\varnothing$. Then, by Remarks 2.2(iii) $d_{0}\left(A_{1}\right)$ and $d_{0}\left(A_{2}\right)$ are two dense subsets in $X$ and consequently, $\mu_{X}^{-1}\left(\mu_{X}\left(A_{1}\right)\right) \cap \mu_{X}^{-1}\left(\mu_{X}\left(A_{2}\right)\right)=\mu_{X}^{-1}\left(\mu_{X}\left(A_{1}\right) \cap \mu_{X}\left(A_{2}\right)\right)=\emptyset$. So, $\mu_{X}\left(A_{1}\right)$ and $\mu_{X}\left(A_{2}\right)$ are two disjoint subsets of $\mathbf{T}_{0}(X)$. Now, according to Lemma 2.5, $X=\overline{d_{0}\left(A_{i}\right)}=\overline{\mu_{X}^{-1}\left(\mu_{X}\left(A_{i}\right)\right)}=\mu_{X}^{-1}\left(\overline{\mu_{X}\left(A_{i}\right)}\right)$, for every $i \in\{1,2\}$. Thus, for every $i \in\{1,2\}, \mu_{X}\left(\mu_{X}^{-1}\left(\overline{\mu_{X}\left(A_{i}\right)}\right)\right)=\overline{\mu_{X}\left(A_{i}\right)}=\mathbf{T}_{0}(X)$, which means that $\mu_{X}\left(A_{1}\right)$ and $\mu_{X}\left(A_{2}\right)$ are two disjoint dense subsets of $\mathbf{T}_{0}(X)$.

Remark 2.6. Clearly, every $\mathbf{T}_{0}$-resolvable space is a resolvable space. The converse does not hold as the following examples show:

(1) Given a set $X$ containing at least two points equipped with the indiscrete topology, it is clear that for, any $x \in X, \overline{\{x\}}=X$, and, consequently, $\mathbf{T}_{0}(X)$ is a one-point space. Now, since any subset of $X$ is dense, $X$ is a resolvable space but $\mathbf{T}_{0}(X)$ is not.

This is a non-trivial space which is resolvable and its $\mathbf{T}_{0}$-reflection is not.

(2) Equip the open interval $X=] 0,1[$ with a topology $\tau$ whose open sets are $\emptyset$, $X$ and the subset $\left.U_{n}=\right] 0,1-\frac{1}{n}[$, for $n \geq 2$. Such a topology is called the Nested Interval Topology. Since every non-empty open set contains both $\frac{1}{8}$ and $\frac{1}{4}, X$ is not $\mathbf{T}_{0}$. That is, $\mathbf{T}_{0}(X) \neq X$. Let, $\left.A_{1}=\right] 0, \frac{1}{4}\left[\right.$ and $A_{2}=\left[\frac{1}{4}, \frac{1}{2}\left[\right.\right.$. Then, $A_{1}$ and $A_{2}$ are disjoint dense subsets. Therefore, $X$ is resolvable. However, $\mathbf{T}_{0}(X)$ is not resolvable. Indeed, we have $\mathbf{T}_{0}(X)=\left\{\operatorname{cl}\left(\frac{1}{4}\right), \operatorname{cl}\left(\frac{1}{2}\right), \operatorname{cl}\left(\frac{2}{3}\right), \operatorname{cl}\left(\frac{3}{4}\right), \operatorname{cl}\left(\frac{4}{5}\right), \operatorname{cl}\left(\frac{5}{6}\right), \ldots\right\}$, where $\left.\operatorname{cl}\left(\frac{1}{4}\right)=\right] 0, \frac{1}{2}\left[, \operatorname{cl}\left(\frac{1}{2}\right)=\left[\frac{1}{2}, \frac{2}{3}\left[, \operatorname{cl}\left(\frac{2}{3}\right)=\left[\frac{2}{3}, \frac{3}{4}\left[, \operatorname{cl}\left(\frac{4}{5}\right)=\left[\frac{4}{5}, \frac{5}{6}\left[, \operatorname{cl}\left(\frac{5}{6}\right)=\right.\right.\right.\right.\right.\right.\right.$ $\left[\frac{5}{6}, \frac{6}{7}\left[, \ldots\right.\right.$ We can see that, if a subset $A$ is dense, then it must contain $\operatorname{cl}\left(\frac{1}{4}\right)$. Hence, $\mathbf{T}_{0}(X)$ does not contain two disjoint dense subsets which makes it an irresolvable space.

Recall that a topological space is said to be sober if any nonempty irreducible closed subset of $X$ has a unique generic point. Let $X$ be a topological space and $\mathbf{S}(X)$ the set of all irreducible closed subsets of $X$ [8]. Let $U$ be an open subset of $X$ and set $\widetilde{U}=\{C \in \mathbf{S}(X): \quad U \cap C \neq \emptyset\}$. Then, the collection $\{\widetilde{U}: \quad U$ is an open subset of $X\}$ provides a topology on $\mathbf{S}(X)$ and the map $\boldsymbol{\eta}_{X}: X \longrightarrow \mathbf{S}(X): x \longrightarrow \overline{\{x\}}$ is a quasihomeomorphism. Moreover, the set $\mathbf{S}(X)$ is a sober space called the sobrification of $X$ and the assignment $\mathbf{S}$ defines a functor from the category Top of topological spaces to itself, see [8]. 
Definition 2.7. Let $X$ be a topological space. $X$ is called $\mathbf{S}$-resolvable space if its sobrification $\mathbf{S}(X)$ is resolvable.

Theorem 2.8. Let $X$ be a topological space. Then, $X$ is an $\boldsymbol{S}$-resolvable space if and only if there exists a family $\mathcal{A}$ of closed irreducible subsets of $X$ such that, for any open set $U$ of $X$, there exist two irreducible subsets $C_{1} \in \mathcal{A}$ and $C_{2} \notin \mathcal{A}$ that both meet $U$.

Proof. Necessary condition. Suppose that $X$ is $\mathbf{S}$-resolvable, then there exists a subset $\mathcal{A}$ of $\mathbf{S}(X)$ such that $\mathcal{A}$ and $\mathbf{S}(X) \backslash \mathcal{A}$ are two disjoint dense subsets of $\mathbf{S}(X)$. Thus, for every open set $U$ of $X, \mathcal{A} \cap \widetilde{U} \neq \emptyset((\mathbf{S}(X) \backslash \mathcal{A}) \cap \widetilde{U} \neq \emptyset)$, which means that there exists $C_{1} \in \mathcal{A}$ such that $C_{1} \cap U \neq \emptyset\left(C_{2} \in(\mathbf{S}(X) \backslash \mathcal{A})\right.$ such that $\left.C_{2} \cap U \neq \emptyset\right)$.

Sufficient condition. It is clear that the family $\mathcal{A}$ defined by the hypothesis satisfies $\overline{\mathcal{A}}=\overline{\mathbf{S}(X) \backslash \mathcal{A}}=\mathbf{S}(X)$. Therefore, $\mathbf{S}(X)$ is a resolvable space.

Examples 2.9. (1) An S-resolvable space need not be resolvable. Indeed, let $X$ be an infinite set and $\alpha \notin X$. Put $Y:=X \cup\{\alpha\}$ and equip $Y$ with the topology whose closed sets are all finite sets of $X$ together with $X$ and $Y$. Clearly, $Y$ is not sober because $X$ is an irreducible closed set of $Y$ without a generic point, so $\overline{\{\alpha\}}=Y$ and $\overline{\{x\}}=\{x\}$, for any $x \in X$.

Now, $\mathbf{S}(X)$ is the subset $Y$ equipped with the topology whose closed sets are all finite sets of $X$ and $Y$.

It is clear that $\{\alpha\}$ is an open set in $Y$, so $Y$ is not resolvable and $Y$ is resolvable since any infinite subset $A$ of $Y$ such that $Y \backslash A$ is also finite satisfies $\bar{A}=\overline{Y \backslash A}=$ $Y$.

(2) A resolvable space need not be an $\mathbf{S}$-resolvable space. Indeed, let $X=\{0,1\}$ be equipped with the indiscrete topology so it is resolvable.

Now, it is clear that $\mathbf{S}(X)$ is a one-point set and, consequently, not resolvable.

\section{FH-Resolvable SPACES AND $\boldsymbol{\rho}$-REsolvable SPACES}

Let $X$ be a topological space. The ring of all real-valued continuous functions will be denoted by $\mathbf{C}(X)$ and $\mathbf{C}^{\star}(X)$ will denote its subring of bounded functions.

Recall that two subsets $A$ and $B$ are said to be completely separated in a topological space $X$ if there exists a mapping $f$ in $\mathbf{C}(X)$ such that $f(a)=0$ for all $a$ in $A$ and $f(b)=1$ for all $b$ in $B$. It will be convenient to say that $x, y \in X$ are completely separated if $\{x\}$ and $\{y\}$ are completely separated. On the one hand, a topological space in which any two distinct points are completely separated is said to be functionally Hausdorff. On the other hand, a topological space is called completely regular (or Tychonoff) if it is $\mathbf{T}_{1}$ and every closed subset $F$ of the space is completely separated from any point $x$ not in $F$. It is clear that a Tychonoff space is functionally Hausdorff and, consequently, a Hausdorff space. Some authors characterize Tychonoff space as a subspace of a compact Hausdorf space but in our case we need the following characterization of Tychonoff spaces.

Theorem 3.1. [13, Proposition 1.7] A space is completely regular if and only if the family of zero-sets of the space is a base for the closed sets (or equivalently, the family of cozero-sets is a base for the open sets). 
Note that $A$ is called a zero-set if there exists an $f \in \mathbf{C}(X)$ such that $A=$ $f^{-1}(\{0\})$. The complement of a zero-set is called a cozero-set.

Now, for a given topological space $X$, we define the equivalence relation $\sim$ on $X$ by $x \sim y$ if and only if $f(x)=f(y)$ for all $f \in \mathbf{C}(X)$. Let us denote by $X / \sim$ the set of equivalence classes and let $\boldsymbol{\rho}_{X}: X \longrightarrow X / \sim$ be the canonical surjection map assigning to each point of $X$ its equivalence class. Since every $f$ in $\mathbf{C}(X)$ is constant on each equivalence class, we can define $\boldsymbol{\rho}(f): X / \sim \longrightarrow \mathbb{R}$ by $\boldsymbol{\rho}(f)\left(\boldsymbol{\rho}_{X}(x)\right)=f(x)$. One may illustrate this situation by the following commutative diagram.

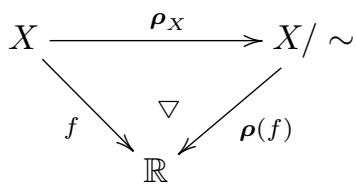

Now, equip $X / \sim$ with the topology whose closed sets are of the form

$$
\cap\left[\boldsymbol{\rho}\left(f_{\alpha}\right)^{-1}\left(F_{\alpha}\right): \alpha \in I\right],
$$

where $f_{\alpha}: X \longrightarrow \mathbb{R}\left(F_{\alpha}\right)$ is a continuous map (a closed subset of $\mathbb{R}$ ). It is well known that, with this topology, $X / \sim$ is a Tychonoff space (see for instance [13]) and it is denoted by $\rho(X)$.

The construction of $\boldsymbol{\rho}(X)$ satisfies some categorical properties:

For each Tychonoff space $Y$ and each continuous map $f: X \longrightarrow Y$, there exists a unique continuous map $\tilde{f}: \boldsymbol{\rho}(X) \longrightarrow Y$ such that $\tilde{f} \circ \boldsymbol{\rho}_{X}=f$. We will say that $\boldsymbol{\rho}(X)$ is the $\boldsymbol{\rho}$-reflection, or the Tychonoff-reflection of $X$.

From the above properties, it is clear that $\rho$ is a covariant functor from the category of topological spaces Top into the full subcategory Tych of Top whose objects are Tychonoff spaces.

On the other hand, the quotient space $X / \sim$ which is denoted by $\mathbf{F H}(X)$ is a functionally Hausdorff space.

The construction $\mathbf{F H}(X)$ satisfies some categorical properties:

For each functionally Hausdorff space $Y$ and each continuous map $f: X \longrightarrow Y$, there exists a unique continuous map $\tilde{f}: \mathbf{F H}(X) \longrightarrow Y$ such that $\tilde{f} \circ \boldsymbol{\rho}_{X}=f$. We will say that $\mathbf{F H}(X)$ is the functionally Hausdorff-reflection of $X$, or the $\mathbf{F H -}$ reflection of $X$.

Consequently, it is clear that $\mathbf{F H}$ is a covariant functor from the category of topological spaces Top into the full subcategory FunHaus of Top whose objects are functionally Hausdorff spaces (for more information see [12]).

Now, as in the first section, we need to recall some notations and results introduced by the authors in [6].

Notations 3.2. [6, Notation 3.1] Let $X$ be a topological space, $a \in X$ and $A$ a subset of $X$. We denote by:

(1) $d_{\boldsymbol{\rho}}(a):=\bigcap\left[f^{-1}(f(\{a\})): f \in \mathbf{C}(X)\right]$.

(2) $d_{\boldsymbol{\rho}}(A):=\cup\left[d_{\boldsymbol{\rho}}(a): a \in A\right]$.

The following results are given in $[6]$.

Proposition 3.3. [6, Proposition 3.2] Let $X$ be a topological space, $a \in X$ and $A$ a subset of $X$. Then: 
(1) $d_{\rho}(A)=\rho_{X}^{-1}\left(\rho_{X}(A)\right)$.

(2) $d_{\rho}(a)$ is a closed subset of $X$.

(3) $A \subseteq d_{\rho}(A) \subseteq \cap\left[f^{-1}(f(A)): f \in \mathbf{C}(X)\right]$.

(4) $\forall f \in \mathbf{C}(X), f(A)=f\left(d_{\rho}(A)\right)$.

Now, we introduce the following definition.

Definition 3.4. Let $X$ be a topological space. $X$ is called a $\boldsymbol{\rho}$-resolvable $\mathbf{F H}$ resolvable) space if its $\boldsymbol{\rho}$-reflection (FH-reflection) is a resolvable space.

In [11, Remark 3.5] it is shown that a closed (open) subset of $\boldsymbol{\rho}(X)$ is of the form $\cap\left[\boldsymbol{\rho}(f)^{-1}(\{0\}): f \in H\right]\left(\cup\left[\boldsymbol{\rho}(f)^{-1}\left(\mathbb{R}^{\star}\right): f \in H\right]\right.$, where $H$ is a collection of continuous maps from $X$ to $\mathbb{R}$.

Now, in order to characterize $\boldsymbol{\rho}$-resolvable spaces and $\mathbf{F H}$-resolvable spaces, we need to recall the following definition introduced in [6].

Definition 3.5. [6, Definition 3.13] Let $X$ be a topological space.

(1) A subset $V$ of $X$ is called a functionally open subset of $X$ ( $F$-open for short) if and only if $d_{\rho}(V)$ is open in $X$.

(2) A subset $V$ of $X$ is called a functionally dense subset of $X$ ( $F$-dense for short) if and only if for any $F$-open subset $W$ of $X, d_{\boldsymbol{\rho}}(V)$ meets $d_{\boldsymbol{\rho}}(W)$.

(3) A nonempty subset $V$ of $X$ is said to be $\boldsymbol{\rho}$-dense, if $g(V) \neq\{0\}$ for every nonzero continuous map $g$ from $X$ to $\mathbb{R}$.

Theorem 3.6. Let $X$ be a topological space. Then, the following statements are equivalent:

(1) $X$ is $\mathbf{F H}-$ resolvable;

(2) There exist two $F$-dense subsets $A_{1}$ and $A_{2}$ of $X$ satisfying $d_{\boldsymbol{\rho}}\left(A_{1}\right) \cap$ $d_{\rho}\left(A_{2}\right)=\emptyset$.

Proof. (1) $\Longrightarrow(2)$

Suppose that $X$ is an $\mathbf{F H}$-resolvable space. Then, there exist two subsets $A_{1}$ and $A_{2}$ of $X$ such that $\boldsymbol{\rho}_{X}\left(A_{1}\right) \cap \boldsymbol{\rho}_{X}\left(A_{2}\right)=\varnothing$ and $\overline{\boldsymbol{\rho}_{X}\left(A_{1}\right)}=\overline{\boldsymbol{\rho}_{X}\left(A_{2}\right)}=\mathbf{F H}(X)$. Thus, $\boldsymbol{\rho}_{X}^{-1}\left(\boldsymbol{\rho}_{X}\left(A_{1}\right) \cap \boldsymbol{\rho}_{X}\left(A_{2}\right)\right)=\boldsymbol{\rho}_{X}^{-1}\left(\boldsymbol{\rho}_{X}\left(A_{1}\right)\right) \cap \boldsymbol{\rho}_{X}^{-1}\left(\boldsymbol{\rho}_{X}\left(A_{2}\right)\right)=\emptyset$, which means that $d_{\boldsymbol{\rho}}\left(A_{1}\right) \cap d_{\boldsymbol{\rho}}\left(A_{2}\right)=\varnothing$. On the other hand, let $U$ be an $F$-open subset of $X$ which means that $\boldsymbol{\rho}_{X}(U)$ is an open set of $\mathbf{F H}(X)$. Since for every $i \in\{1,2\}, \boldsymbol{\rho}_{X}\left(A_{i}\right)$ is a dense subset of $\mathbf{F H}(X)$, then $\boldsymbol{\rho}_{X}(U) \cap \boldsymbol{\rho}_{X}\left(A_{i}\right) \neq \varnothing$. Therefore, by applying $\rho_{X}^{-1}$, we get easily, $d_{\boldsymbol{\rho}}(U) \cap d_{\boldsymbol{\rho}}\left(A_{i}\right)=\emptyset$, for every $i \in\{1,2\}$ and, consequently, $A_{1}$ and $A_{2}$ are two $F$-dense subsets of $X$ such that $d_{\rho}\left(A_{1}\right) \cap d_{\rho}\left(A_{2}\right)=\emptyset$.

$(2) \Longrightarrow(1)$

Conversely, let $A_{1}$ and $A_{2}$ be two $F$-dense subsets of $X$ satisfying $d_{\boldsymbol{\rho}}\left(A_{1}\right) \cap$ $d_{\boldsymbol{\rho}}\left(A_{2}\right)=\emptyset$. For any open subset $\rho_{X}(V)$ of $\mathbf{F H}(X)$, where $V$ is a subset of $X$, we have $V$ is an $F$-open subset of $X$ and, thus, $d_{\rho}\left(A_{1}\right)\left(d_{\rho}\left(A_{2}\right)\right)$ neessarily meets $\rho_{X}(V)$. Thus, $\left.\boldsymbol{\rho}_{X}^{-1}\left(\boldsymbol{\rho}_{X}\left(A_{1}\right)\right) \cap \boldsymbol{\rho}_{X}^{-1}\left(\boldsymbol{\rho}_{X}(V)\right)\right)$ and $\left.\boldsymbol{\rho}_{X}^{-1}\left(\boldsymbol{\rho}_{X}\left(A_{2}\right)\right) \cap \boldsymbol{\rho}_{X}^{-1}\left(\boldsymbol{\rho}_{X}(V)\right)\right)$ are nonempty subsets, which implies that $\boldsymbol{\rho}_{X}(U) \cap \boldsymbol{\rho}_{X}\left(A_{1}\right)$ and $\boldsymbol{\rho}_{X}(U) \cap \boldsymbol{\rho}_{X}\left(A_{2}\right)$ are also nonempty. Hence, $\boldsymbol{\rho}_{X}\left(A_{1}\right)$ and $\boldsymbol{\rho}_{X}\left(A_{2}\right)$ are two dense subset of $\mathbf{F H}(X)$.

Moreover, $\boldsymbol{\rho}_{X}^{-1}\left(\boldsymbol{\rho}_{X}\left(A_{1}\right) \cap \boldsymbol{\rho}_{X}\left(A_{2}\right)\right)=\boldsymbol{\rho}_{X}^{-1}\left(\boldsymbol{\rho}_{X}\left(A_{1}\right)\right) \cap \boldsymbol{\rho}_{X}^{-1}\left(\boldsymbol{\rho}_{X}\left(A_{2}\right)\right)=d_{\boldsymbol{\rho}}\left(A_{1}\right) \cap$ $d_{\boldsymbol{\rho}}\left(A_{2}\right)=\emptyset$. Therefore, $\boldsymbol{\rho}_{X}\left(A_{1}\right)$ and $\boldsymbol{\rho}_{X}\left(A_{2}\right)$ are disjoint sets. 
By noting that, for any subset $A$ of $X, d_{\rho}\left(d_{\rho}(A)\right)=d_{\rho}(A)$, the following corollary is immediate

Corollary 3.7. Let $X$ be a topological space. Then, the following statements are equivalent:

(1) $X$ is $\mathbf{F H}$-resolvable;

(2) There exist two disjoint $F$-dense subsets $d_{\boldsymbol{\rho}}\left(A_{1}\right)$ and $d_{\boldsymbol{\rho}}\left(A_{2}\right)$ of $X$.

Proposition 3.8. [6, Proposition 3.4] Let $X$ be a topological space and A a subset of $X$. Then, the following statements are equivalent:

(i) $A$ is a $\boldsymbol{\rho}$-dense subset of $X$;

(ii) $\boldsymbol{\rho}_{X}(A)$ is a dense subset of $\boldsymbol{\rho}(X)$.

Theorem 3.9. Let $X$ be a topological space. Then, the following statements are equivalent:

(1) $X$ is $\boldsymbol{\rho}$-resolvable;

(2) There exist two $\boldsymbol{\rho}$-dense subsets $A_{1}$ and $A_{2}$ of $X$ such that $d_{\boldsymbol{\rho}}\left(A_{1}\right)$ and $d_{\boldsymbol{\rho}}\left(A_{2}\right)$ are disjoint.

Proof. (1) $\Longrightarrow(2)$

Since $X$ is a $\rho$-resolvable space, there exist two subsets $A_{1}$ and $A_{2}$ of $X$ such that $\boldsymbol{\rho}_{X}\left(A_{1}\right) \cap \boldsymbol{\rho}_{X}\left(A_{2}\right)=\varnothing$ and $\overline{\boldsymbol{\rho}_{X}\left(A_{1}\right)}=\overline{\boldsymbol{\rho}_{X}\left(A_{2}\right)}=\boldsymbol{\rho}(X)$. In the same way as in Theorem 3.6, one can easily see that $d_{\boldsymbol{\rho}}\left(A_{1}\right)$ and $d_{\boldsymbol{\rho}}\left(A_{2}\right)$ are disjoint subsets of $X$. Now, the $\rho$-density of $A_{1}$ and $A_{2}$ are immediate by Proposition 3.8.

$(2) \Longrightarrow(1)$ Conversely, let $A_{1}$ and $A_{2}$ be two $\rho$-dense subsets of $X$ such that $d_{\boldsymbol{\rho}}\left(A_{1}\right) \cap d_{\boldsymbol{\rho}}\left(A_{2}\right)=\emptyset$.

Clearly, by Proposition 3.8, $\left(\boldsymbol{\rho}_{X}\left(A_{1}\right)\right.$ and $\left(\boldsymbol{\rho}_{X}\left(A_{2}\right)\right.$ are two dense subsets of $\boldsymbol{\rho}(X)$. Now, since $d_{\rho}\left(A_{1}\right) \cap d_{\rho}\left(A_{2}\right)=\rho_{X}^{-1}\left(\rho_{X}\left(A_{1}\right)\right) \cap \rho_{X}^{-1}\left(\rho_{X}\left(A_{2}\right)\right)=\rho_{X}^{-1}\left(\rho_{X}\left(A_{1}\right) \cap\right.$ $\left.\rho_{X}\left(A_{2}\right)\right)=\emptyset$, then $\rho_{X}\left(A_{1}\right) \cap \rho_{X}\left(A_{2}\right)=\emptyset$. Therefore, $\boldsymbol{\rho}_{X}\left(A_{1}\right)$ and $\boldsymbol{\rho}_{X}\left(A_{2}\right)$ are two disjoint dense subsets of $\rho(X)$ and consequently $\rho(X)$ is resolvable or equivalently $X$ is $\boldsymbol{\rho}$-resolvable.

In the same way, the following result is immediate.

Corollary 3.10. Let $X$ be a topological space. Then, the following statements are equivalent:

(1) $X$ is $\boldsymbol{\rho}$-resolvable;

(2) There exist two disjoint $\boldsymbol{\rho}$-dense subsets $d_{\boldsymbol{\rho}}\left(A_{1}\right)$ and $d_{\boldsymbol{\rho}}\left(A_{2}\right)$ of $X$.

Remark 3.11. If $X$ is an $\mathbf{F H}$-resolvable space, then $X$ is a $\boldsymbol{\rho}$-resolvable space.

Indeed, let $X$ be an FH-resolvable space. Then, there exist two subsets $A_{1}$ and $A_{2}$ of $X$ such that $d_{\boldsymbol{\rho}}\left(A_{1}\right)$ and $d_{\boldsymbol{\rho}}\left(A_{2}\right)$ are disjoint $F$-dense subsets. By $[6$, Remark 3.3 ] every $F$-dense subset is a $\boldsymbol{\rho}$-dense subset, thus, according to the last theorem, $X$ is a $\boldsymbol{\rho}$-resolvable space.

Acknowledgement. The authors are very grateful to the referee for careful reading of the paper and for useful comments. 


\section{REFERENCES}

[1] M. Al-Hajri and K. Belaid, Resolvable spaces and compactifications, Advances in Pure Mathematics 3 (2013), 365-367.

[2] A. V. Arhangel'skii and P. J. Collins, On submaximal spaces, Topology Appl. 64 (1995), 219-241.

[3] K. Belaid and L. Dridi, I-spaces, nodec spaces and compactifications, Topology Appl. 161 (2014), 196-205.

[4] K. Belaid, L. Dridi and O. Echi, Submaximal and door compactifications, Topology Appl. 158 (2011), 1969-1975.

[5] K. Belaid, O. Echi and S. Lazaar, $T_{(\alpha, \beta)}$-spaces and the Wallman compactification, Int. J. Math. Math. Sci. 2004 (2004), 3717-3735.

[6] L. Dridi, S. Lazaar and T. Turki, F-door spaces and F-submaximal spaces, Appl. Gen. Topol. 14 (2013), 97-113.

[7] L. Dridi, A. Mhemdi and T. Turki, F-nodec spaces, Appl. Gen. Topol. 16 (2015), 53-64.

[8] A. Grothendieck and J. Dieudonné, Éléments de Géométrie Algébrique, Springer-Verlag, Heidelberg, 1971.

[9] A. Grothendieck and J. Dieudonné, Éléments de Géométrie Algébrique I: Le Langage des Schemas, Inst. Hautes Études Sci. Publ. Math. No. 4, 1960.

[10] E. Hewitt, A problem of set-theoretic topology, Duke Math. J. 10 (1943), 309-333.

[11] O. Echi and S. Lazaar, Reflective subcategories, Tychonoff spaces, and spectral spaces, Topol. Proc. 34 (2009), 307-319.

[12] S. Lazaar, On functionally Hausdorff spaces, Missouri J. Math. Sci. 25 (2013), 88-97.

[13] R. C. Walker, The Stone-Čech Compactification, Springer-Verlag, Berlin, 1974.

Intissar Dahane, Faculty of Sciences of Tunis, University of Tunis El Manar, Tunisia e-mail: intissardahane@gmail.com

Lobna Dridi, Department of Mathematics, Tunis Preparatory Engineering Institute, University of Tunis, 1089 Tunis, Tunisia

e-mail: lobna_dridi_2006@yahoo.fr

Sami Lazaar, Faculty of Sciences of Tunis, University of Tunis El Manar, Tunisia e-mail: salazaar72@yahoo.fr 